\section{LETTERS TO THE EDITOR}

\section{Diagnosis of PCP in HIV seropositive patients}

We read with interest the article by Sauleda and colleagues on the use of a simplified exercise test for the initial differential diagnosis of Pneumocystis carinii pneumonia (PCP) in HIV positive patients (February 1994;49:112-4) and would like to make the following points. Firstly, the risk factors for the acquisition of HIV infection were not given other than to state that 36 of the patients were intravenous drug users. It was not clear how these 36 patients divided between the two groups. Intravenous drug use is associated with impaired lung function independently of acquired infection and, in addition, intravenous drug users are more susceptible to pyogenic infections. Of the initial 60 subjects enrolled in the study $25 \%$ were excluded, but it was not stated how many were excluded because of pre-existing respiratory disease that could alter the $\mathrm{SaO}_{2}$. No data were presented on the smoking history of the subjects. Nieman et al have shown that cigarette smokers develop PCP more rapidly than non-smokers although initial lung function, as measured by spirometry and carbon monoxide transfer factor (TLCO), was not statistically different between the two study groups. ${ }^{1}$ Data were also missing on the PCP prophylaxis used by patients in the study; previous studies have shown that this can have a significant effect on the diagnosis of PCP through the decreased yield with bronchoalveolar lavage.

A specificity value of $91 \%$ compares very favourably with other studies using exercise oximetry $^{2}$ and we wonder if the less standardised exercise protocol with a shorter duration of activity allowed the patients who were more ill to complete the test.

Like the authors, we are committed to the use of non-invasive tests for the diagnosis of PCP. We favour measurement of TLCO as a screening test, as exercise tests have been shown in the past to be poorly tolerated. ${ }^{2} \mathrm{At}$ our unit a TLCO value below $70 \%$ has been shown to have a sensitivity of $92 \%$ and a specificity of $72 \%$ for diagnosis of acute PCP. ${ }^{3}$ These results are comparable with those in this study but, like the authors, we proceed promptly to bronchoscopy, initiating treatment early if appropriate.

\section{CJ SKINNER} RJ COKER

DM MITCHELI St Mary's Hospital, London W2 $1 N Y$ $U K$

1 Nieman RB, Fleming J, Coker RJ, Harris JRW, Mitchell DM. The effect of cigarette smoking on the development of AIDS in HIV-1-seropositive individuals. AIDS 1993,7:705-10.

2 Smith DE, McLuckie A, Wyatt J, Gazzard B. Severe exercise hypoxemia with normal or near normal X-rays: a feature of Pneumocystis carinii infection. Lancet 1988;ii:1049-51.

3 Shaw RJ, Roussak C, Forster SM, Harris JRW, Pinching AJ, Mitchell DM. Lung function abnormalites in patients infected with the abnormalities in patients infected with the human inmunodeficiency virus with and without overt
AUTHORS' REPLY We find the comments from the St Mary's group interesting and agree with most of their observations but there are several points that we would like to clarify.

With regard to factors that could have had a potential influence on the results, as risk factors for HIV were very similar in both groups (PCP, 16 intravenous drug abusers (IDA) and six male homosexuals; non-PCP, 20 IDA, one homosexual and two heterosexual partners of HIV seropositive) we felt this did not modify the results. On the other hand, although many patients were smokers, the mean (SD) age in both groups (31(1) and 28(1) years, respectively), as well as the absence of pre-existing pulmonary diseases, allowed us to assume that their lung function was not seriously affected by these factors. Finally, none of the patients had previously received prophylactic treatment for PCP. Many of the subjects were only HIV seropositive without any previous opportunistic infection.

We agree that classical lung function tests (principally TLCO) can be useful for screening purposes in these patients. However, they have important "logistic" limitations as the devices required for classical exercise tests or for the measurement of TLCO are expensive and untransportable, they are not available 24 hours a day, need qualified technicians, and cannot be performed in the emergency department (or at the patient's home). Our own experience in both the lung function laboratory (during morning periods) and the emergency department (afternoon and night, once a week, with limited technology available) compelled us to look for a simplified screening test. This test had to be easy to perform and able to be analysed in a short period of time. Thus, inessential factors - such as the use of a cycloergometer or predetermined levels of charge, and the measurement of oxygen uptake or carbon dioxide production should be excluded. We felt, and still feel that, where possible, physiopathological knowledge should be applied in a simple way for clinical purposes.

J GEA

J SAULEDA JM BROQUETAS Servei de Pneumologia, Hospital del Mar Barcelona, Spain

\section{Setting up a pulmonary rehabilitation programme}

I read with great interest the article by Clark (March 1994;49:270-8) as the Department of Thoracic Medicine at the London Chest Hospital has recently begun to provide a programme of pulmonary rehabilitation. The paper contained many points that were found to be important during the initiation of our own service.

As a physiotherapist I feel encouraged by the prospect of a more involved and fulfilling role with patients disabled by chronic lung disease, as time constraints often prevent this holistic approach on a busy ward.

Dr Clark suggests that the involvement of physiotherapists in pulmonary rehabilitation will require them to undergo further training as their background in exercise physiology may be limited. However, exercise physiology is a subject covered in great detail during our training, with Gray's Anatomy being the student physiotherapist's bible. When applying our training to the practice of pulmonary rehabilitation, careful analysed thought is therefore ensured. Designing and carrying out exercise programmes for all ages and disabilities is also an integral part of our training, and we are certainly well familiar with the particular difficulties of patients with COPD. Although it is suggested that physiotherapists should attend leisure centre classes for experience in learning about exercise, our extensive training provides us with a comprehensive knowledge of the physiological and practical basis of exercise therapy applicable to chronically disabled patients.

I hope this letter will add to the information required by chest physicians on some of the subjects we cover during training, and will reinforce the view that physiotherapists should be included in a pulmonary rehabilitation programme.

RINA GARNHAM

Pulmonary Rehabilitation Programme, Department of Thoracic Medicine, London Chest Hospital, London E2 97X,

\section{BOOK NOTICES}

Prevention of Respiratory Diseases. A Hirsch, M Goldberg, J-P Martin, R Masse. (Pp 744; \$185.00). New York: Marcel Dekker, 1993. 0824788508.

This is number 68 in the influential "Lung Biology in Health and Disease" series. The book's editors are French and half the chapters come from French groups, the others coming from the USA, Canada, Holland, Italy and the UK. Only two of the 58 contributors are based in the UK, which may reflect the low priority prevention currently has in British respiratory medicine within our current health care system and in academic medicine.

The book takes a broad view of the prevention of respiratory disease, dividing it into four parts: occupational diseases, environmental sources of respiratory diseases, biological markers, and tobacco. The part on respiratory disease related to tobacco is, rightly, over one third of the book. It is particularly valuable as it brings together 11 contributions from several disciplines. It is possible to highlight only a few in this review. Kauffmann and her colleagues review the relationship between bronchial responsiveness and smoking and include useful tables summarising the epidemiological evidence. This chapter is typical of the high quality of many in the book - wide ranging, authoritative, well referenced, and carefully argued. In stylistic contrast was the chapter on theories of smoking behaviour which was illuminating because strategies to prevent smoking must operate within a conceptual framework. The authors discuss smoking as 
an addiction, as learned behaviour, as a volitional act, and as a social phenomenon.

The role of occupation is regrettably often neglected and the reviewer was glad to see almost a third of the book devoted to nine chapters covering occupational cancers, asthma, non-specific lung disease, epidemiological topics, and the measurement of occupational exposures. The succinct and clear account of occupational asthma covers the issues relevant to prevention, but it would have been good to see a separate chapter focusing on primary preventive strategies by controlling exposure in the workplace. It is possible that occupational asthma and rhinitis are more common than occupational respiratory cancer, although the balance in this section does not reflect this with five of the nine chapters being on cancer.

Samet and Spengler review the prevention of diseases due to indoor and outdoor air pollution in a readable overview which is a good introduction to the section on environmental sources of respiratory disease. Other chapters cover indoor radon, the air conditioned building, indoor allergens, and the problem of concurrent exposure to multiple pollutants. The section on biological markers has an introduction to the subject of genetic markers in complex diseases and also covers topics related to emphysema.

Infection is the major omission. The book would have benefitted from tighter editing, with less overlap and a more consistent structure for the chapters. It lacked a general introduction to the concepts of primary prevention (control of harmful exposures), secondary prevention (early detection of disease), and tertiary prevention (action to minimise complications and disability). This is a book that all but the smallest libraries should buy, and that many individuals will wish to purchase for themselves. $-\mathrm{KV}$.

The Pulmonary Artery Catheter Methodology and Clinical Applications 2nd Edition. Charles L Sprung. (Pp 295). USA: Critical Care Research Associates, 1993. 0963731505.

I was doing "rounds" on a Los Angeles Intensive Care Unit with an old friend who had trained in Britain but had emigrated to California. The resident was presenting the cases of those patients who had been admitted overnight. The first case he described as follows: "A 49 year old Caucasian male was admitted as an emergency last night with a wedge pressure of $32 \mathrm{~mm} \mathrm{Hg}$." My friend and I looked at each other aghast. Has clinical medicine taken such a second place to technology that we were not to be treated to the history and examination before the pulmonary artery catheter had been inserted?

This story illustrates the movement in medicine towards high technology, particularly in the USA. Nowhere is this movement more apparent than in the vast increase in monitoring systems for the critically ill and not so ill. It is estimated that in the USA critical care consumes about $15 \%$ of the total health budget. Important in these costs is the increasing use of pulmonary artery catheters. It is estimated that approximately one million pulmonary artery catheters are used every year in the US at a cost of two billion dollars. Their use is clearly increasing. In $19757 \cdot 2 \%$ of patients with acute myocardial infarction had catheters inserted and in 1984 this had risen to almost $20 \%$.

Clearly these figures are nothing like as high in the UK and Europe, but even here there is increasing reliance on the numbers produced by catheter rather than careful clinical observation. It now appears that the pulmonary artery catheter is so well entrenched in US coronary care units and ICUs that it is impossible to do a proper trial of its use. Indeed, no proper trial has ever been done into the effectiveness of these catheters despite their widespread use. Since the pulmonary artery flotation catheter is here to stay and is unlikely to be replaced in the short term by non-invasive instrumentation, it is very important that the catheter is only used where the information gained will make a significant change to the management of the patient, and is only placed by competent personnel trained in its insertion and in the interpretation of its findings.

This is the second edition of Charles Sprung's book which aims to ensure that these objectives are achieved. Indeed the illustration on the frontispiece is of a simple balance with a stethoscope on one side and a flotation catheter on the other. The balance must always be between clinical judgement and invasive measurement. The authors take great pains to point out that the catheter should only be used in the proper circumstances. Indeed, the authors take on an almost paranoid air in defending the use of the catheter from its significant critics, especially Professor Eugene Robin who has claimed that not only is the pulmonary artery catheter useless but in many cases it is actually positively dangerous. Dr Sprung, an anaesthetist and critical care physician, has amassed an international group of authors who come mostly from the USA, but also from Israel, France, and Belgium.

The book is divided into three sections. The first section is methodological where the indications for pulmonary artery catheterisation, techniques of insertion, and complications are discussed in detail. This for me was the most useful section of the book because it described in a clear, easy to read fashion many of the rules of right heart catheterisation that should be followed by all those involved. I was surprised, however, at the list of indications which included myocardial infarction where there is hypotension, pulmonary oedema, mitral regurgitation, tamponade, or ventricular septal defect. It also included pulmonary thromboembolism, congestive cardiac failure, shock, respiratory failure in the face of myocardial disease, and many states which could be described as problems of intravascular volume such as renal disease, cirrhosis, and burns. I think many physicians would take exception to using a pulmonary artery catheter for these indications where a central venous pressure line would suffice and would probably be less dangerous. The main indication for right heart catheterisation is for measurement of the two variables that cannot be obtained clinically - left atrial pressure and pulmonary artery pressure - that would make a significant difference to the management of the patient. This is particularly true, for example, in right ventricular infarction where inexperienced physicians might treat a high IVP with diuretics only to reduce left ventricular preload to unacceptable levels. It may also be true in a patient with respiratory failure where there is widespread lung shadowing and the question is raised whether or not this is due to myocardial dysfunction or to a so called low pressure pulmonary oedema. In many other conditions such as burns, renal disease, and pulmonary thromboembolism there is very little added benefit of a pulmonary artery catheter over a right atrial line.

The second section of the book is on clinical applications, particularly the measurements and calculations derived from them. The third section covers new techniques such as extravascular lung water measurement, right ventricular ejection fraction, and comparison of non-invasive echocardiography with direct measurements from a pulmonary artery catheter. The final chapter consists of 13 clinical studies where the use of a catheter made a significant difference to the management of individual patients. These examples are very useful and make clear much of the more theoretical material encountered earlier in the book.

This book is really for use in the USA where huge numbers of pulmonary artery catheters are placed. In the UK it would be welcome in intensive care units and, perhaps, coronary care units where junior doctors involved in right heart catheterisation need easy access to a description of the best of clinical practice.-AJP

\section{NOTICE}

\section{9th International Conference on Lung Sounds}

The 19th International Conference on Lung Sounds will be held at the Rappaport Institute, Technion, Haifa, Israel on 28-30 September 1994. For further information please contact Raymond L H Murphy, Attn: Barbara Keith, Faulkner Hospital, Pulmonary Department, 1153 Centre St, Boston, Massachusetts 02130, USA. Telephone: (617) 522 5800, ext 1968 or Fax: (617) 5248663. 